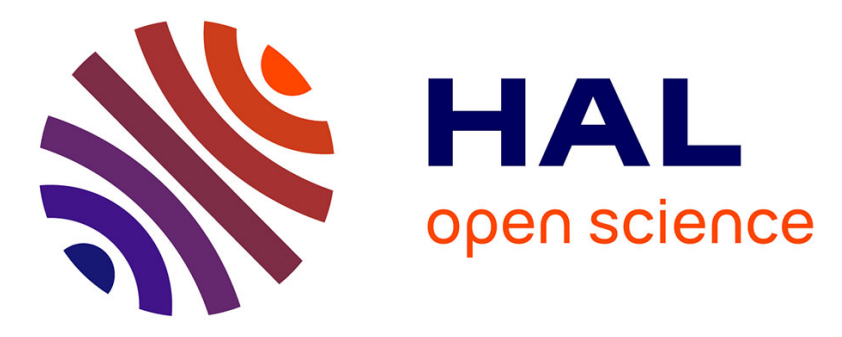

\title{
Approach for the 3D-Alignment in Micro- and Nano-scale Assembly Processes
}

Thomas Wich, Christian Stolle, Manuel Mikczinski, Sergej Fatikow

\section{To cite this version:}

Thomas Wich, Christian Stolle, Manuel Mikczinski, Sergej Fatikow. Approach for the 3D-Alignment in Micro- and Nano-scale Assembly Processes. 5th IFIP WG 5.5 International Precision Assembly Seminar (IPAS), Feb 2010, Chamonix, France. pp.167-173, 10.1007/978-3-642-11598-1_19 . hal01055688

\section{HAL Id: hal-01055688 \\ https://hal.inria.fr/hal-01055688}

Submitted on 13 Aug 2014

HAL is a multi-disciplinary open access archive for the deposit and dissemination of scientific research documents, whether they are published or not. The documents may come from teaching and research institutions in France or abroad, or from public or private research centers.
L'archive ouverte pluridisciplinaire HAL, est destinée au dépôt et à la diffusion de documents scientifiques de niveau recherche, publiés ou non, émanant des établissements d'enseignement et de recherche français ou étrangers, des laboratoires publics ou privés.

\section{(c)(1)}

Distributed under a Creative Commons Attribution| 4.0 International License 


\title{
Approach for the 3D-alignment in micro- and nano-scale assembly processes
}

\author{
Thomas Wich ${ }^{1,2}$, Christian Stolle ${ }^{1}$, Manuel Mikczinski ${ }^{2}$, Sergej Fatikow ${ }^{1,2}$ \\ ${ }^{1}$ University of Oldenburg, Division Microrobotics and Control Engineering, D-26111 \\ Oldenburg, Germany \\ ${ }^{2}$ OFFIS Institute for Information Technology, Technology Cluster Automated Nanohandling, \\ Escherweg 2, D-26121 Oldenburg, Germany \\ \{Thomas.Wich, Christian.Stolle, Manuel.Mikczinski, Fatikow\}@Uni-Oldenburg.de
}

\begin{abstract}
Most assembly processes on the nano-scale take place in a Scanning Electron Microscope (SEM) for the reason of high magnification range of the microscope itself. Like all microscopes, the SEM delivers visual data just in two dimensions. This is a bottleneck for all assembly processes which require of course information of the parts to join in a third dimension. This paper shows an approach with a dedicated sensor. As an example for an assembly process a carbon nano tube (CNT) is fixed on a sharp metal tip. The sensor used detects contact between these two parts by exciting a bimorph cantilever made from piezoelectric material. It is shown that with this approach the contact is reliably detected. Recent experiments on introducing a new excitation structure show the possibility to add more dimensional testing in the same way as the one dimensional type.
\end{abstract}

Keywords: Assembly process, carbon nano tube, TouchDown sensor, automation.

\section{Introduction}

The deterministic assembly of parts and components on the micro- and nano-scale is derived from assembly processes as known from the macro- and meso-scale. Although a lot of similarities exist for both regimes, micro- and nano-scale assembly faces some distinct differences, making it a challenging task both for manual and automated execution [8], [2].

Fig. 1 shows a typical flowchart for such an assembly process, which consist of the tasks Transport, Adjustment, Joining, Separation and Inspection. For the assembly of one product, these tasks have to be repeated in a sequence. In contrary to self- and parallel assembly, 
usually these tasks are not parallelised, but repeated $n$ times for the production of $\mathrm{n}$ products.

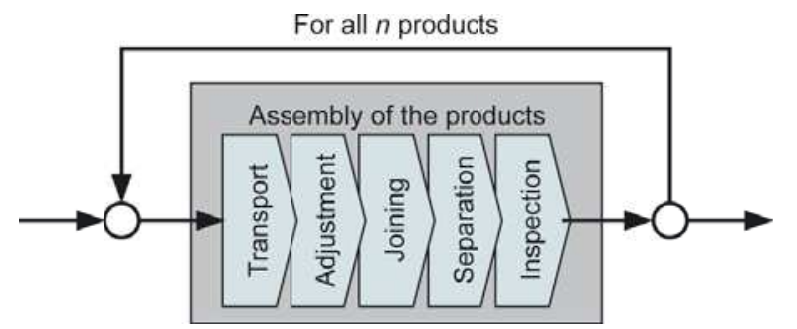

Fig. 1. Process layout for the automated serial assembly on the nano-scale.

The transport of the parts consumed during assembly usually involves several orders of magnitude in geometrical dimensions, e.g. actuator travel in the millimetre regime to a final position with nanometre accuracy is involved. This is mainly due to the fact, that micro- or nano-scale parts are often mounted on meso-scale parts. In automated assembly, these are fed to the assembly machine on adaptors [11], which have dimensions controllable by human operators. In the adjustment task, two parts or even a part and the handling tool (e.g. a gripper) are adjusted relative to each other in position and orientation. This has to be done in six dimensions, however, depending on the part to be handled, some simplifications can be used (e.g. for spherical objects). However, the most important outcome of this task is the defined contact between the parts, which is a key prerequisite for the forthcoming joining. The joining task is the key task within the process chain, as two parts are united to one product here. With respect to micro- and nano-scale assembly, the number of available techniques is very limited, simply because not all macro- and meso-scale techniques can be down-scaled in a straight forward way. On the micro- and nanoscale, parts tend to stick to each other [5], to the underlying substrate and to the handling tools due to adhesive surface forces. To enable the automation of parts handling, they need to be stored on the adaptor in a well-defined state, i.e. with defined distances in between, separately and held in place. On the nano-scale, the latter one is e.g. achieved by growing parts on a substrate [7], resulting in a strong chemical or physical bond in between. Thus, for cutting the parts from the substrate or even separating them from the handling tool, a dedicated separation task is necessary. The inspection task serves as a measure of quality of 
the assembly process and usually involves testing of the bond between the parts.

Especially in the transportation and in the adjustment tasks, the 3Dalignment of parts or tool and part, respectively, is challenging for the above mentioned reasons. In section 2, these challenges will be described in more detail, resulting from the characteristics of the magnifying vision sensors. Based on typical sceneries in nano-scale assembly, the methods for in- and out of-plane coarse alignment will be discussed shortly. Additionally, the implemented approach for coarse alignment is discussed in more detail. Section 3 describes the TouchDown-sensor, a tool used for contact detection between tool and substrate or part, respectively. The results of the implemented alignment process will be described section 4 in more detail. Section 5 concludes the approach.

\section{Challenges and approaches in aligning tool and object}

Magnifying vision sensors are literally spoken the gate for accessing the micro- and nano-scale. Most commonly, optical light microscopes are used for the micro-scale; for accessing the nano-scale, scanning electron microscopes (SEM) and its derivates or - less frequently scanning probe microscopes are used. Within this paper, the focus will be on SEM [1], [10]. Although they provide practical image acquisition speed, high and adjustable magnification, the high depth of focus and parasitic effects like image drift limit their applicability as a precise and unique global sensor [6].

The high depth of focus, usually considered as a benefit for analytical purposes, requires an experienced operator in manual assembly and dedicated sensor tools or methods in automated assembly, respectively, for out of plane alignment of part and tool. The parasitic effects, resulting from hysteresis effects on the scanning coils of the SEM and from charging effects in the scene, complicate the in-plane alignment. Therefore, the approach between tool and object is realised using visual servoing [9], [4], i.e. the distance between both objects is measured with object recognition methods in the recorded image. These relative measurements allow for precise alignment, however, the procedure requires continuous image acquisition, which limits the process speed compared to open-loop-alignment based on a world model. 
The out-of-plane alignment is achieved by a combination of two methods. For coarse alignment, a series of images is taken at different focus distance [3]. This focus series is evaluated for sharpness in regions of interests (RoI), drawn around the tool and the part, resulting in two distinct curves. By comparing the sharpness-over-focus curves, the distance between tool and object is evaluated by the distance between the sharpness peaks for both RoIs. However, due to the high depth-of-focus of the SEM, the precision of this method is limited to several $\mu \mathrm{m}$ in the necessary magnification. Consequently, for final alignment, the tool and object are adjusted to each other until they are in mechanical contact. The contact is measured by the TouchDownsensor. As an application example, we will follow the in- and out-of plane alignment sequence between a CNT and a fine etched metal tip further. Fig. 2 shows the starting scenery.

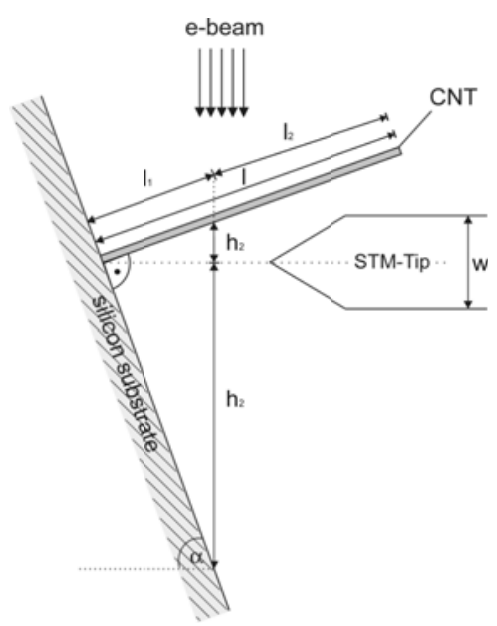

Fig. 2. Side view of the scene for the assembly of CNTs. The CNT is grown on a silicon substrate, which is mounted with a tilt angle in the SEM. The CNT is approached by a fine etched metal tip. A possible contact needs to be detected in all three dimensions.

\section{Contact detection with the TouchDown-sensor}

The TouchDown sensor is a critical part in the alignment sequence, as the mechanical contact between tool and part has to be detected by measuring the contact force, as the SEM lacks the third dimension. Generally, three configurations are suitable for the implementation of such a sensor. At first, the senor can be integrated into the tool. As the 
handling tools for nano-scale assembly are fabricated in silicon batch processes, such integration is costly and the flexibility is low. Secondly, the tool can be mounted on the sensor, or as a third solution, the adaptor carrying the parts can be mounted on the sensor. The latter two approaches allow for highly flexible configurations, which are quasi independent of the tools or parts used for the assembly process. On the downside, a high sensitivity is necessary for such an approach. The initial TouchDown-sensor is basically a piezo-bimorph cantilever: two piezoelectric layers are connected by a glass fibre substrate (Fig. 3). The upper one is used as actuator, whereas the lower one is used as receiver. This tactile sensor has been implemented as a vibrating sensor, oscillating at its resonance frequency with small amplitude [11]. The occurring contact between tool and part changes the oscillation characteristics, modelled as an additional damping element. The change in phase shift between the driving and the driven oscillation, which is measured with a lock-in amplifier, is strongly dependent on the force exerted on the sensor. A more-dimensional approach under development detects contact with the same principle. But it is designed to superimpose this sensing principle on a second movement (e.g. a circular movement) with lower frequency but higher amplitude.
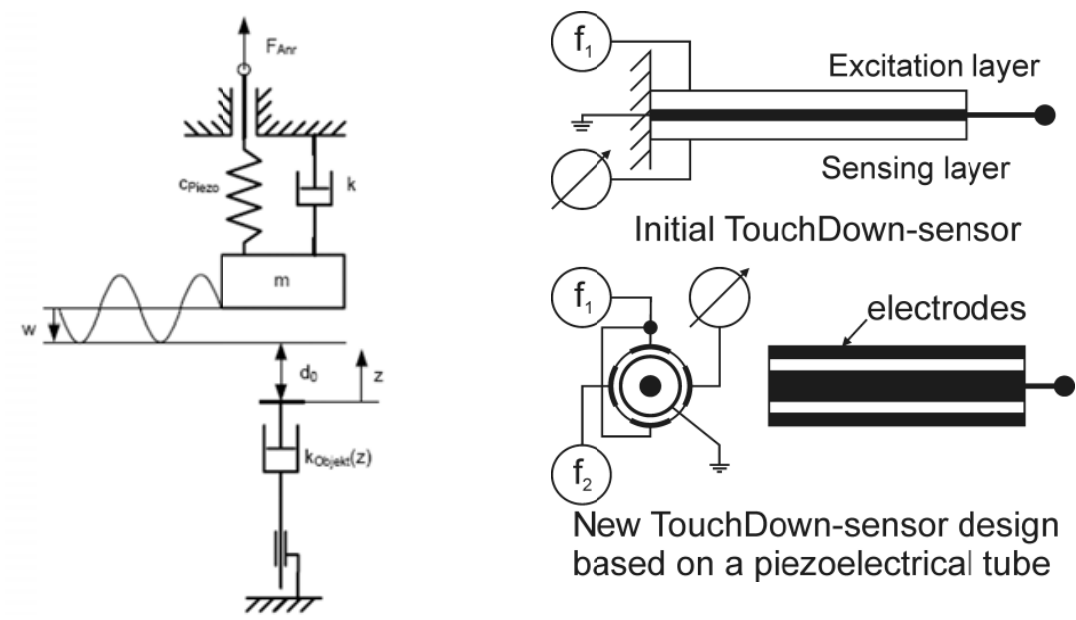

Fig. 3. Mechanical model for the TouchDown-sensor, modeled as a spring-mass-damper system. The contact between the object, e.g. CNT, is modeled as a second damper (left). The mechanical setup for both approaches is schematically depicted (right). 


\section{$4 \quad$ Results}

The automated alignment sequence consisted of three sub-sequences. The first step (Fig. 4a) consisted of the course x,y-alignment at a scan field size of $35 \mu \mathrm{m}$. The z-course alignment was performed by depthfrom-focus (cp. section 2). The second step was the fine alignment step in $\mathrm{x}$-direction (Fig. 4b). The $\mathrm{x}$ alignment has been separated from the $\mathrm{y}$ alignment in order to get more reliable results from visual servoing by avoiding overlap. Finally, the metal tip moved below the CNT (Fig. 4c). The steps 2 and 3 could be performed safely because of a distance of more than $25 \mu \mathrm{m}$ between substrate and CNT (cp. Fig. $2 \mathrm{~h} 1$ and h2). Starting from the $\mathrm{x}-\mathrm{y}$ aligned setup the touchdown sequence has been executed.

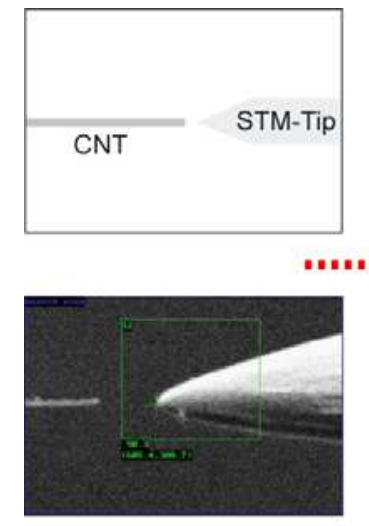

(a)

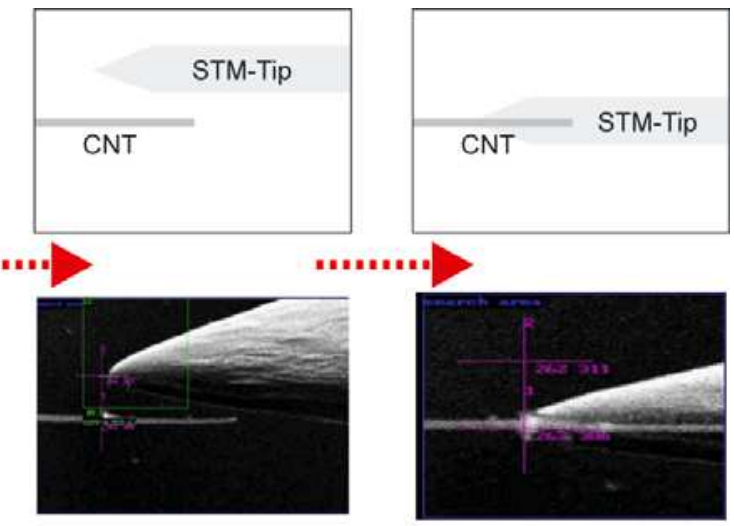

(b)

(c)

Fig. 4. Alignment sequence between a fine etched metal tip and a CNT. The top row shows the schematic drawings, the bottom row the corresponding images taken in the SEM.

Fig. 5 (left) shows the measured oscillation amplitude at resonance frequency in $\mathrm{nm}$ over the amplitude of the driving voltage in $\mathrm{mV}$. Obviously, a wide range of amplitudes can be set with relatively small voltages. It is important that the oscillation amplitude of the sensor is smaller than the critical size of the object (CNT). This allows for even smaller CNTs or other nano-scale objects to be detected with the TouchDown-sensor. 

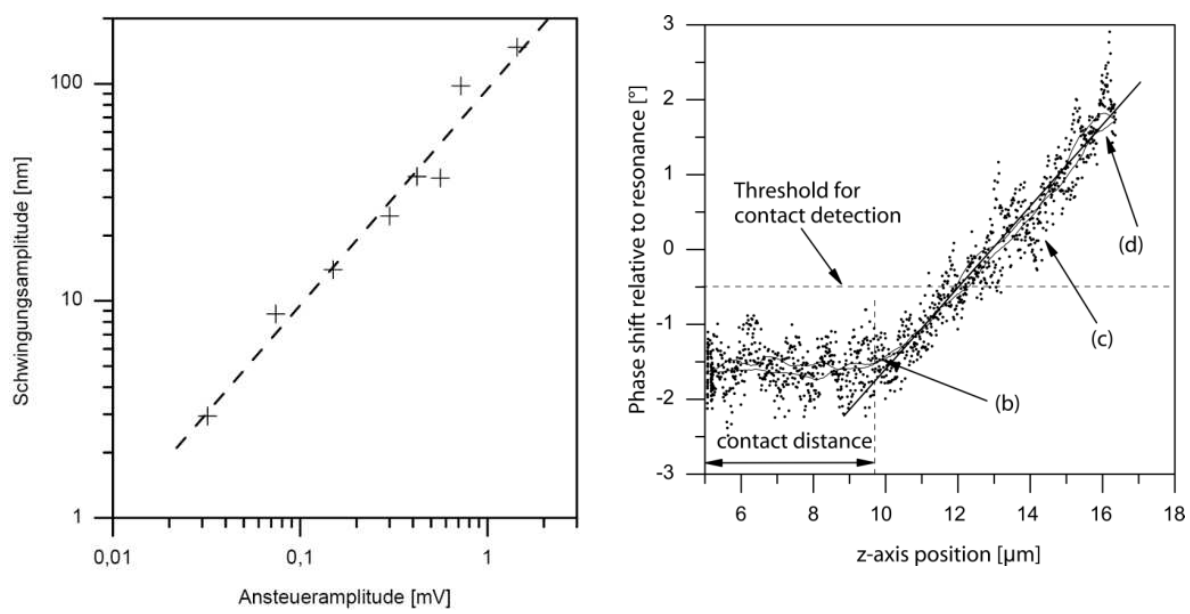

Fig. 5. Oscillation amplitude of the TouchDown-sensor in its working point over the amplitude of the driving voltage (left). Contact measurements between a fine etched metal tip and a CNT (right).

Figure 5 (right) shows the contact detection between a fine etched metal tip and a CNT. The contact is detected as soon as a predefined relative phase shift threshold has been reached. The sensor was excited with a voltage of $0.7 \mathrm{mV}$. This corresponds to oscillation amplitude of $98 \mathrm{~nm}$. The result indicates that the contact can be safely detected. The measured data showed quasi linear behaviour after the first contact with a factor of $7.135 \cdot 10^{3} \mathrm{~s} / \mathrm{m}$. The force applied to the CNT at detection threshold was about $4.9 \mu N$.

The reliability of the contact detection method has been evaluated during 500 touchdown cycles. The metal-tip started at a distance of $4 \mu \mathrm{m}$ below the CNT at the beginning of each cycle and moved at a velocity of $0.133 \mu \mathrm{m} / \mathrm{s}$ towards the CNT. The phase shift threshold has been set to $1.5^{\circ}$ for contact detection. During the $8.3 \mathrm{~h}$ of measurement $98.4 \%$ of all touchdowns have been detected correctly.

\section{Conclusion}

The 3D-alignment procedure in an assembly process on the nanoscale in SEM consists of in- and out-of plane alignment steps between tool and part. Both steps are crucial for automated assembly on the nanoscale and compared to the macro- and meso-scale, dedicated tools and 
methods have to be developed. The TouchDown-sensor provides the necessary contact detection. Its reliability was proven in this presentation. Three-dimensional measurement would add extra value to a possible implementation of automated assembly systems. This will be achieved in further research by superimposing the TouchDownprinciple on an advanced structure which is capable of more dimensional movement.

\section{References}

[1] Automatic nanohandling station inside a scanning electron microscope. 222(1):117-128, 2008.

[2] J. Cecil, Derek Powell, and Daniel Vasquez. Assembly and manipulation of micro devices-a state of the art survey. Robot. Comput.-Integr. Manuf., 23(5):580-588, 2007.

[3] Volkmar Eichhorn, Sergej Fatikow, Thomas Wich, Christian Dahmen, Torsten Sievers, Karin Andersen, Kenneth Carlson, and Peter Bøggild. Depth-detection methods for microgripper based cnt manipulation in a scanning electron microscope. Journal of Micro Nano Mechatronics.

[4]S. Fatikow, J. Seyfried, S. Fahlbusch, A. Buerkle, and F. Schmoeckel. A Flexible Microrobot-Based Microassembly Station. Journal of Intelligent and Robotic Systems, 27(12):135-169, 2000.

[5]Ronald S. Fearing and $\mathrm{Kg}$ The. Survey of sticking effects for micro parts handling. In In IEEE/RSJ Int. Workshop on Intelligent Robots \& Systems (IROS, pages 212-217, 1995.

[6]T. Sievers. Global sensor feedback for automatic nanohandling inside a scanning electron microscope. In Proceedings of IPROMS NoE Virtual International Conference on Intelligent Production Machines and Systems, pages 289-294, 2006. Received the Best Presentation Award.

[7]K. B. K. Teo, M. Chhowalla, G. A. J. Amaratunga, W. I. Milne, D. G. Hasko, G. Pirio, P. Legagneux, F. Wyczisk, and D. Pribat. Uniform patterned growth of carbon nanotubes without surface carbon. Applied Physics Letters, 79:1534-+, September 2001.

[8]Christian Dahmen Tim Luttermann Oliver Frick Mirko Naroska Sergej Fatikow Thomas Wich, Christian Stolle. Zunami: Automated assembly processes on the nanoscale. In 4M/ICOMM 2009 - The Global Conference on Micro Manufacture, pages 81-85, 2009.

[9]B. Vikramaditya and B.J. Nelson. Visually guided microassembly using optical microscopes and active vision techniques. Proceeding of the 1997 IEEE International Conference on Robotics and Automation, 1997.

[10] T. Wich and H. Hülsen. Automated Nanohandling by Microrobots, chapter Robotbased Automated Nanohandling. Springer Verlag, 2008.

[11] Thomas Wich. Tools and methods for the automation of serial nano-assembly processes in the Scanning Electron Microscope. (Werkzeuge und Methoden zur Automatisierung der seriellen Nanomontage im Rasterelektronenmikroskop). Logos Verlag Berlin GmbH, 2008. 